\title{
Zum Gedenken an Bartel Leendert van der Waerden (2.2.1903-12.1.1996)
}

Günther Frei

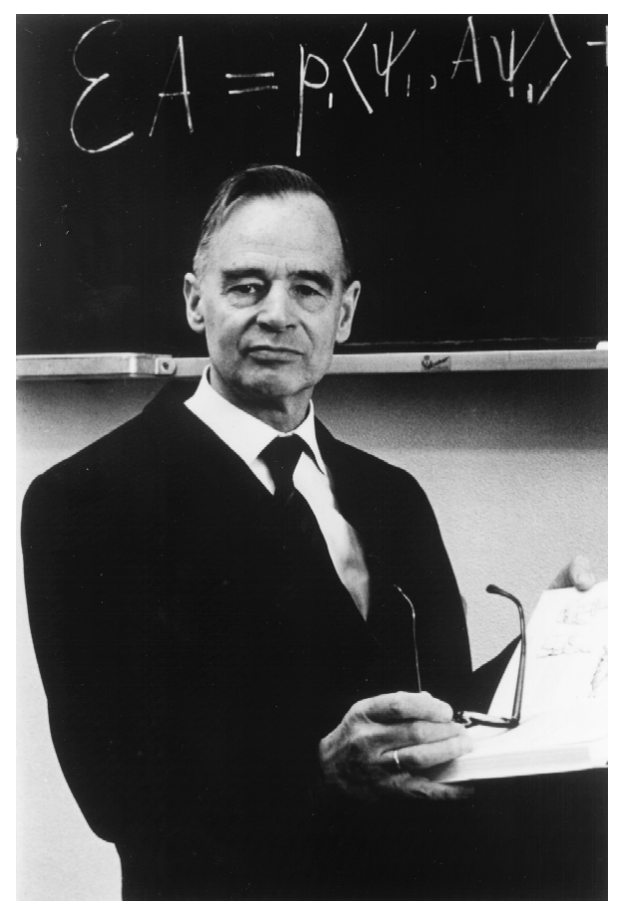

Am 12. Januar 1996 ist mit dem Hinscheiden von Bartel Leendert van der Waerden ein aussergewöhnliches Gelehrtenleben zu Ende gegangen. Van der Waerden gehörte zu den bedeutendsten Mathematikern des 20. Jahrhunderts, und er war vielleicht der letzte Gelehrte, der nicht nur alle Gebiete der Mathematik überschauen, sondern sie auch mit bedeutenden Beiträgen bereichern konnte. Seine wissenschaftlichen Arbeiten gehen sogar weit über die Mathematik hinaus, betreffen sie doch auch die Physik, die Biologie, die Musik, die Philosophie und die Geschichte der Mathematik und der Naturwissenschaften, insbesondere der Astronomie und der Physik. In breiteren Kreisen ist van der 
Waerden durch sein epochemachendes zweibändiges Werk "Algebra" (Springer, Grundlehren, 1930, damals mit dem Titel "Moderne Algebra") bekannt geworden. Mehrere Generationen von Mathematikern und Physikern haben daraus die moderne abstrakte Algebra - aufgebaut auf Axiome und die daraus folgenden grundlegenden Strukturen von Gruppen, Ringen und Körpern - kennengelernt, wie sie von R. Dedekind, H.Weber, D. Hilbert, E. Steinitz, E. Artin und Emmy Noether geschaffen worden war. Auch heute noch, nach fast 70 Jahren, sind die Bücher und Vorlesungen an den Universitäten über höhere Algebra im wesentlichen der Darstellung von van der Waerden verpflichtet.

Dem Leser der "Neuen Zürcher Zeitung" ist van der Waerden vertraut durch die Reihe von wohlfundierten Beiträgen zu Platon, Pythagoras, den Pythagoreern, über die Perserkönige und die indische Astronomie (1966-1972) und dem Leser der "Elemente der Mathematik" auch wegen der schönen Arbeiten über Polygone mit maximalem Flächeninhalt (1950), über Einfall und Überlegung in der Mathematik (1953, 1954), über die Einführung des Logarithmus im Schulunterricht (1957), über klassische und moderne Axiomatik (1967), über einen Satz über räumliche Fünfecke (1970), zum Andenken an Andreas Speiser (1971) und über die Wechselwirkung von Mathematik und Physik (1973). Auf den schweizerischen Schulunterricht hat van der Waerden durch seine regelmässig gehaltene Vorlesung "Mathematik für Naturwissenschafter" einen unschätzbar grossen Einfluss ausgeübt, war doch diese Vorlesung nicht nur für Naturwissenschafter, sondern auch für angehende Sekundarlehrer aus verschiedenen Kantonen Pflichtfach. Damit stellte van der Waerden sicher, dass exaktes Denken in breitesten Kreisen bis hinab zur Sekundarschule Eingang fand. Sein Engagement um die Vermittlung eines kompetenten Mathematikunterrichtes schon auf der Stufe der Mittelschulen hat er auch durch seine Thesen zur Reform des Mathematikunterrichtes, veröffentlicht im Organ des Vereins Schweizerischer Mathematik- und Physiklehrer (1966), unter Beweis gestellt. In Zürich hat er viele Diplomanden und über vierzig Doktoranden auf den verschiedensten Gebieten ausgebildet, die an Hoch- und Mittelschulen seine Ideen und seine Auffassung der Mathematik als Wissenschaft weiter verbreitet haben. Auf seine Schüler und auf jeden, der mit ihm in Kontakt kam, machte er durch seine aussergewöhnliche Universalität, seine Strenge im logischen Denken, seine Vorstellungskraft und insbesondere durch seine überaus schnelle Erfassung komplizierter Zusammenhänge einen ungeheuren Eindruck.

Ich erinnere mich an einen Vortrag eines Kommilitonen über eine Arbeit von Singer im Geometrie-Seminar. Nachdem jener einen Hauptsatz ausgesprochen hatte, unterbrach ihn van der Waerden mit dem Einwand, dass dieser Satz von Singer nicht korrekt sein könne. Zehn Minuten später, als der Vortragende sich angeschickt hatte, den Beweis vorzutragen, unterbrach ihn van der Waerden wieder mit den Worten: "Ich habe jetzt ein Gegenbeispiel". Van der Waerdens Geist war immer in Bewegung. Sein Kollege der Theoretischen Physik an der Universität Zürich, Walter Heitler, nannte van der Waerden den eindruckvollsten Wissenschafter, dem er je begegnet sei. Sein Kollege Heinz Hopf an der ETH, selbst einer der bedeutendsten Mathematiker unseres Jahrhunderts, hielt in seinem Gutachten anlässlich der Berufung van der Waerdens an die Universität Zürich im Jahre 1951 fest: "B. L. van der Waerden ist einer derjenigen Mathematiker, die während der letzten 25 Jahre eine wesentliche Änderung im Aussehen der Mathematik herbeigeführt haben. Bestimmt wäre niemand besser als van der Waerden geeignet, an 
der Universität eine neue 'algebraische Schule' zu gründen." Von Dieudonné, einem der Begründer der Bourbaki-Gruppe, wissen wir, dass das Lehrbuch "Moderne Algebra" von van der Waerden diese Gruppe ganz entscheidend geprägt hat und damit auch die von dieser Gruppe ausgegangene, die ganze Mathematik während längerer Zeit dominierende Entwicklung, die auf der strukturellen Auffassung der Mathematik beruht.

Hauptarbeitsgebiet von van der Waerden war die Algebraische Geometrie, jenes Forschungsgebiet, das aus der Theorie der algebraischen Kurven hervorgegangen ist und das heute im Brennpunkt der mathematischen Forschung steht, weil sich dort Algebra, Geometrie, Zahlentheorie und Analysis aufs engste und geheimnisvollste begegnen. Dieses Gebiet spielt nicht nur in der aktuellen Mathematik, z.B. in der Arithmetischen Geometrie, sondern auch in der modernen physikalischen Forschung eine hervorragende Rolle, so etwa in der Theorie der Elementarteilchen und auch im Bereich der Relativitätstheorie. Die moderne Algebraische Geometrie hat in der Tat mit den grundlegenden Arbeiten von van der Waerden ihren Anfang genommen. Van der Waerden war es, der ihr in den 20er Jahren mit strengen Begriffen und Beweisen eine solide Basis gegeben hat. Bedeutende Beiträge lieferte van der Waerden auch zur Topologie, Gruppentheorie, Zahlentheorie, Analysis, Statistik und Physik. Darüber hinaus hat sich van der Waerden, insbesondere durch Arbeiten in seinen späteren Jahren, also während der Zürcher Zeit, um die Geschichte der Wissenschaften, hauptsächlich der antiken Mathematik und Astronomie, bedeutende Verdienste erworben. Ihr hat er weit über 100 Einzelarbeiten und sechs Bücher gewidmet. Als einer der ganz wenigen Mathematikhistoriker, die auch in der mathematischen Wissenschaft selbst in hohem Masse schöpferisch tätig waren, hat er der Wissenschaftsgeschichte ganz neue Impulse geben können.

Bartel Leendert van der Waerden wurde am 2. Februar 1903 in Amsterdam geboren. Erste Anregungen zur Mathematik erhielt er von seinem Vater, der Ingenieurwissenschaften studiert hatte und dann an der Oberrealschule in Amsterdam Mathematik unterrichtete, später einem grossen Elektrizitätswerk vorstand und dann als sozialistischer Politiker dem Landesparlament angehörte. Nach einem erfolgreichen und rasch durchlaufenen Studium der Mathematik und Physik an der Universität Amsterdam, wo L. E. J. Brouwer, G. Mannoury, H. de Vries und R. Weitzenböck seine Lehrer waren, erlaubte ihm sein Vater - nach erfolgreich bestandenem Schlussexamen bei de Vries im Herbst 1924 - ein weiteres Semester in Göttingen zu verbringen, damals das wissenschaftliche Zentrum für mathematische Forschung weltweit. Dort lernte van der Waerden Topologie von Hellmuth Kneser und Methoden der Mathematischen Physik bei Courant und seinen Schülern Lewy und Friedrichs. Am meisten geprägt aber hat ihn Emmy Noether mit ihren Arbeiten und Vorlesungen zur abstrakten Algebra, ihren Hinweisen auf die dazugehörige Literatur und ihrer Betonung der strukturellen Auffassung der Mathematik. Damit war es van der Waerden möglich, die auf intuitiven Vorstellungen beruhenden und daher ungenauen geometrischen Definitionen und Sätze von Schubert zur abzählenden Geometrie, die er schon in Amsterdam in Vorlesungen bei de Vries kennengelernt hatte, und jene der italienischen Geometer Severi, Castelnuovo und Enriques zur Algebraischen Geometrie erstmals mit den modernen algebraischen Begriffen der Körper- und Ringtheorie scharf und präzise zu fassen. So entstanden noch vor dem eigentlichen Abschluss des Studiums bedeutende Arbeiten über Invariantentheorie, über die Nullstellentheorie der 
Polynomideale und über den Multiplizitätsbegriff in der Algebraischen Geometrie, die in den "Mathematischen Annalen" veröffentlicht wurden. Daher konnte sich van der Waerden in seiner während der Militärdienstzeit geschriebenen Dissertation - sie durfte der Universität Amsterdam nur auf Latein oder Holländisch eingereicht werden - darauf beschränken, nur Resultate ohne Beweise zu präsentieren. Sie trägt den Titel "De algebraiese grondslagen der meetkunde van het aantal" und stellt eine Art Programmschrift zu den Grundlagen des Schubertschen Kalküls der abzählenden Geometrie und weit darüber hinaus zu der Algebraischen Geometrie ganz allgemein dar. Er reichte sie am 26. März 1926 ein, formal als Schüler von Hendrik de Vries. Anschliessend ermöglichte ihm ein auf Empfehlung von R. Courant und Emmy Noether hin erteiltes Rockefeller-Stipendium je ein weiteres Studiensemester in Göttingen und eines bei Artin in Hamburg. Dort entstand das bereits erwähnte Lehrbuch zur modernen Algebra, das auf Vorlesungen von Artin beruht und das ursprünglich als Gemeinschaftswerk mit Artin geplant war. (Als van der Waerden sehr schnell und erfolgreich mit der Abfassung voranschritt, hatte ihm Artin die Redaktion ganz überlassen.) Darin sind auch die neuen Ideen und Sätze von Emmy Noether sowie seine eigenen mitverarbeitet.

1927 habilitierte sich van der Waerden in Göttingen, und schon 1928 erhielt er einen Ruf nach Rostock. Jedoch gab er dem gleichzeitig erfolgten Ruf nach Groningen den Vorzug, wo durch die Berufung von J. A. Barrau nach Utrecht eine Stelle vakant geworden war. Diese war ihm von Barrau schon zu seiner Schulzeit an der Höheren Bürgerschule in Amsterdam versprochen worden, nachdem er als junger Schüler Barrau auf Ungenauigkeiten und Fehler in dessen Buch über Analytische Geometrie aufmerksam gemacht hatte. Drei Jahre nach dem am 6. Mai 1928 ergangenen Ruf als Ordinarius nach Groningen erfolgte die Ernennung zum Professor und Mitdirektor des Mathematischen Seminars und des Mathematischen Institutes an der Universität Leipzig, wobei die Anwesenheit der beiden Physiker Werner Heisenberg und Friedrich Hund und die seines Landsmannes Peter Debye besonders attraktiv wirkten. Dort hat van der Waerden sogleich auch am Seminar Heisenberg-Hund teilgenommen und sich die Quantenmechanik sehr schnell zu eigen gemacht. Aus dieser Mitarbeit ist das Buch "Die gruppentheoretische Methode in der Quantenmechanik" hervorgegangen, das lange Zeit für die Physiker das eigentliche Referenzwerk darstellte. In Leipzig machte van der Waerden auch die Bekanntschaft des Philosophen Hans-Georg Gadamer, dessen Vorlesungen über Plato seinem Interesse für die Philosophie und Mathematik der Griechen neuen Auftrieb gab. Inzwischen hatte van der Waerden Camilla Rellich, die Schwester des bekannten Mathematikers Franz Rellich, geheiratet, die er noch in Göttingen anlässlich eines weiteren Aufenthaltes im Sommer 1929 kennengelernt hatte. Ihr offenes, lebensfreudiges Wesen verband sich auf ideale Weise mit van der Waerdens eher zurückhaltender Art. So war denn auch die Ehe ausserordentlich glücklich und harmonisch und nicht zuletzt auch mitverantwortlich für van der Waerdens so erfolgreiches Wirken. Aus dieser Ehe sind drei Kinder hervorgegangen.

Vor und während des Krieges war van der Waerden in Leipzig als Ausländer, der sich stets geweigert hatte, seine holländische Staatsangehörigkeit aufzugeben, vielen Anfeindungen von Seiten der Nazis ausgesetzt. Dies konnte ihn aber in seiner integren Haltung nicht beirren. Mit Heisenberg und Hund und dem Physikochemiker Karl Bonhoeffer wehrte er sich für die vom politischen System Verfolgten sowie gegen die Gleichschal- 
tung an den Universitäten. 1984 hat die damalige Karl-Marx Universität in Leipzig seine grossen Verdienste um diese Universität, auch gerade wegen dieser seiner Haltung, mit der Verleihung des Titels eines Ehrendoktors gewürdigt. Das war nur eine der vielen Ehren, die dem grossen Gelehrten im Laufe der Zeit zuteil geworden sind. Noch am Tage seines Todes hat ihn die Sächsische Akademie der Wissenschaften zu ihrem Ehrenmitglied ernannt.

Bei den vielen Bombenangriffen auf Leipzig verlor van der Waerden nicht nur Hab und Gut, sondern auch seine wissenschaftlichen Unterlagen und seine gesamte Korrespondenz. Die darauf folgende Zeit verbrachte die Familie zum Teil bei der Schwiegermutter in Graz und dann, nach dem Kriegsende, in Laren in Holland im Haus seiner Eltern. Dank der Hilfe seines Freundes Hans Freudenthal erhielt van der Waerden eine Stelle bei Shell in Amsterdam, wo er sich mit Problemen der praktischen und angewandten Mathematik, etwa der Optimierungstheorie, beschäftigte. 1947 folgte eine Gastprofessur an der Johns Hopkins Universität in Baltimore (USA), wo man ihm eine Professur anbot, die er aber ablehnte. 1948 entschied sich van der Waerden für den ihm offerierten Lehrstuhl an der Universität in Amsterdam. Obgleich es sich dabei um eine sehr vorteilhafte Stellung handelte, nahm er 1951 den Ruf an die Stelle des verstorbenen Rudolf Fueter als Professor für Mathematik und als Direktor des Mathematischen Institutes an die Universität Zürich an. Dieser Hochschule ist er bis an sein Lebensende treu geblieben, obwohl er auch darnach noch Rufe ins Ausland erhielt, so etwa 1953 nach München.

Mit seinem grossen und uneigennützigen Einsatz zum Wohle der Wissenschaft und der Öffentlichkeit in Forschung und Lehre auf allen Stufen hat van der Waerden nicht nur der Schweiz, sondern weit über sie hinaus unschätzbare Dienste geleistet. Als Herausgeber der berühmten "gelben" Sammlung "Grundlehren der mathematischen Wissenschaften" bei Julius Springer sowie der Zeitschriften "Mathematische Annalen" und "Archive for History of Exact Sciences" setzte er der mathematisch-naturwissenschaftlichen Forschungsgemeinschaft weltweit hohe Massstäbe.

Nach seiner Emeritierung im Jahre 1971 hat van der Waerden seine reichhaltige Bibliothek dem von ihm angeregten und neu geschaffenen Institut für Geschichte der Mathematik vermacht und diesem durch eine lange Reihe bis ins hohe Alter verfasster historischer Arbeiten zu weltweitem Ansehen verholfen. Es gibt wohl kaum einen geeigneteren Ort für ein solches Institut als Zürich, das sich auf eine so reiche und lange mathematische Tradition berufen kann. Darüber hinaus hatte van der Waerden schon vor einigen Jahren seinen wissenschaftlichen Nachlass der Wissenschaftshistorischen Sammlung der ETH-Bibliothek übergeben.

Ein Verzeichnis der Veröffentlichungen van der Waerdens hat sein Schüler und späterer Kollege Herbert Gross in den "Elementen der Mathematik" (Band 28, 1973, 26-32) publiziert. Diese Liste ist später in den von F. Hirzebruch herausgegebenen "Selected Papers" (Springer-Verlag, 1983) und in der Zeitschrift "Nieuw Archief voor Wiskunde" ((4) 12, 1994, 179-193) ergänzt worden.

Eine ausführlichere Würdigung mit vollständigem Werkverzeichnis ist vom Autor für die Jahresberichte der Deutschen Mathematiker-Vereinigung geplant. Es ist auch zu hoffen, 
dass alle Einzelarbeiten van der Waerdens, die so viele neue und verschiedenartige Forschungsergebnisse enthalten, bald in den gesammelten Werken vereinigt werden können.

Eine Würdigung der einzigartigen Persönlichkeit van der Waerdens wäre nicht vollständig, wenn man seiner nicht auch als eines im eigentlichen und wahren Sinne grossen Humanisten gedenken würde. Es gilt auch für ihn, was er über die von ihm so sehr verehrte Lehrmeisterin Emmy Noether in einem in Graz und Heidelberg gehaltenen Vortrag gesagt hat: "Sie war durch und durch ein guter Mensch, frei von jedem Egoismus, frei von aller Eitelkeit, frei von Pose, und sie half immer jedem Menschen, wo sie konnte." Nie hat man aus seinem Munde Abschätziges über Kollegen vernommen. Wo immer er konnte, hat er seine Schüler angespornt und gefördert auf der Suche nach Neuem, nach wissenschaftlicher Klarheit und letztlich auf der Suche nach über die Wissenschaft hinausweisender Weisheit. Nichts könnte seine Persönlichkeit besser beschreiben als die Worte seines Sohnes Hans van der Waerden anlässlich der Abdankungsfeier am 19. Januar 1996: "Sokrates war das Vorbild, dem mein Vater nacheiferte, der grosse Meister, in dessen Fussstapfen er wandelte, wo immer er sich aufhielt. Sokrates: in seinem unsäglich anspruchslosen Lebenswandel, seiner Geringschätzung alles dessen, was der persönlichen Eitelkeit schmeichelt, seiner Gleichgültigkeit auch gegenüber vielen Freuden des Leibes, seiner unerbittlichen Suche aber nach Erkenntnis und Wahrheit, und auch nach Gerechtigkeit."

\section{Günther Frei}

Département de mathématiques et de statistique

Université Laval

Ste-Foy, Québec, G1K 7P4

Canada 